Bei allen anderen Verbindungen hat sich unterschiedlicher Fremdstoffgehalt nur in einer Veränderung der Absorptionskonstanten bemerkbar gemacht, die Lage der Absorptionskante jedoch nicht merklich beeinflußt.

Tab. 1 gibt eine Zusammenstellung der wichtigsten Daten der untersuchten Verbindungen und die optisch ermittelte Breite der verbotenen Zone im Vergleich $\mathrm{zu}$ den durch elektrische Messungen gewonnenen Werten*.

Die Breite der verbotenen Zone läßt sich auf etwa $5 \%$ genau bestimmen, während der Meßfehler des Reflexionsvermögens und damit des Brechungsindex etwa $3 \%( \pm 0,1)$ beträgt. Die Werte für die Absorptionskonstante können noch nicht als stoffspezifisch angesehen werden, da sie wesentlich vom Fremdstoffgehalt der Proben abhängen, über den noch nichts Näheres ausgesagt werden kann. Die in dieser Tabelle angegebenen elektrisch

* Nach freundlicher brieflicher Mitteilung von Hrn. Hrostowski haben H. B. Briggs, C. F. Cunnings, H. J. Hrostowski u. M. Tanenbaum in den Bell Tel. Laboratories die Brechungsindizes von AlSb, GaAs, GaSb, InAs und InSb nach der Methode von H. B. Briggs im Infraroten bestimmt, außerdem die Durchlässigkeit und die Breite der verbotenen Zonen. Es besteht im wesentlichen Übereinstimmung mit den in vorliegender Arbeit angegebenen Werten. und optisch bestimmten Werte der Bandbreite sind nicht miteinander vergleichbar, da erstere unter Voraussetzung einer linearen Temperaturabhängigkeit die Breite der verbotenen Zone am absoluten Nullpunkt angeben, die optischen Werte dagegen bei Zimmertemperatur gewonnen sind. Zum Zusammenschluß der Werte muß erst der Temperaturkoeffizient des Bandabstandes bestimmt werden, wozu Messungen bei tiefen Temperaturen in Vorbereitung sind. An Indiumantimonid wurden solche Messungen von Tanen baum und Maita ${ }^{24}$ durchgeführt und führten zu einer Übereinstimmung der Werte unter der Annahme einer effektiven Masse der Ladungsträger von 0,083 Elektronenmassen.

Den Herren O. G. Folberth, R. Grimm und H. J. Schell danken wir für die Überlassung der Proben.

${ }^{21}$ H. Welker, Z. Naturforschg. 8a, 248 [1953].

22 O. G. Folberth, R. Grimm u. H. Weiß, Z. Naturforschg. 8a, 826 [1953].

${ }_{23}$ O. Madelung u. H. Wei $B$, Z. Naturforschg. 9a, 527 [1954].

24 M. Tanenbaum u. J. P. Maita, Phys. Rev. 91, 1009 [1953].

\title{
Über die Lumineszenz in hochpolymeren Substanzen. I
}

\author{
Von H. Hin Richs \\ Aus dem Physikalischen Institut der Justus-Liebig-Hochschule Gießen \\ (Z. Naturforschg. 9a, 617-624 [1954]; eingegangen am 5. März 1954)
}

\begin{abstract}
Es wurde bei der Einlagerung von Anthracen in Polystyrol festgestellt, daß die Lumineszenz des eingebetteten Fluoreszenzstoffes wesentlich davon abhängig ist, ob der Zusatz vor oder nach der Polymerisation erfolgte. Im ersten Fall ergibt sich bei dem größten Teil der eingelagerten Moleküle eine vom normalen Lösungszustand (letzterer Fall) abweichende stärkere Wechselwirkung zwischen dem Fluoreszenzstoff und dem Hochpolymer. Die Wellenzahl des Elektronenübergangs sinkt, während die Schwingungsterme des Grundzustandes unverändert bleiben. Die Erniedrigung der Wellenzahl wurde auch bei der Kathodenstrahlanregung beobachtet. Vermutlich tritt sowohl bei der Polymerisation wie auch bei der Kathodenstrahlanregung eine Verbindung von Anthracen mit Polystyrol-Radikalen ein, wobei die Anthracen-Ringstruktur erhalten bleibt.
\end{abstract}

$\mathrm{K}$ unststoff-Folien mit Fluoreszenzstoff-Einlagerung sind für Szintillations- und Dosismeßzwecke von Interesse. Von besonderem Vorteil sind ihre leichte Handhabe und einfache Herstellung. Weiterhin kann man ihre Form dem jeweiligen Zweck bequem anpassen. Über die Wechselwirkung von energiereicher Strahlung mit fluoreszierenden Kunststoffen liegen wenig Beobachtungen vor. In der vorliegenden Arbeit wird das Verhalten der
Fluoreszenz einiger organischer Leuchtstoffe in Polystyrol untersucht.

Im folgenden ersten Teil werden die Veränderungen der Fluoreszenz von Anthracen bei der Einlagerung unter der Einwirkung von UV - und Kathodenstrahlanregung mitgeteilt. In dem nachfolgenden II. Teil sollen die Ergebnisse bei der Kathodenstrahlanregung von Polystyrolfolien mit anderen Leuchtstoffeinlagerungen besprochen werden. 


\section{Versuchsanordnung}

Die Kunststoffpräparate wurden mit $50 \mathrm{kV}$ Kathodenstrahlen im Vakuum bestrahlt. Abb. 1 zeigt die benutzte Apparatur. Um mehrere Präparate ohne Belüftung der Anlage nacheinander bestrahlen zu können, wurde der Präparathalter drehbar angeordnet und durch Simmer-Ringe abgedichtet. Die Fluoreszenzintensität wurde mit einem Sekundärelektronenverstärker vom Typ 1 P 21 und einem Multiflexgalvanometer gemessen. Die Messung des Elektronenstroms erfolgte durch Vergleich des Gesamtelektronenstroms,

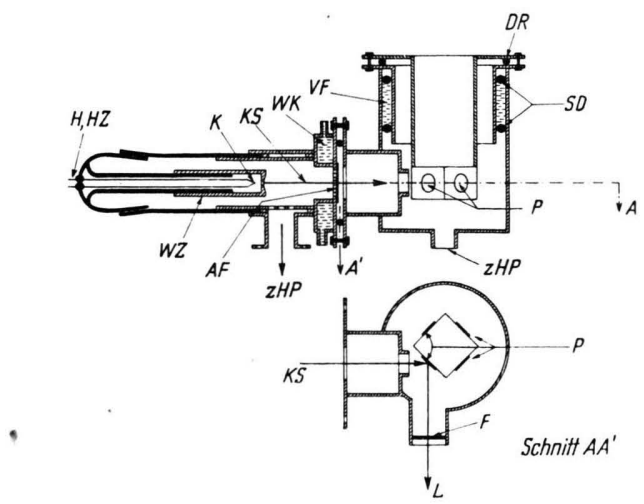

Abb. 1. Versuchanordnung. KS Kathodenstrahl, WK Wasserkühlung, VF Vakuumfett, DR Dichtungsring, $\mathrm{H}$, HZ Heizung und Hochspannungszuführung, K Kathode, SD Simmer-Ring-Dichtung, WZ Wehnelt-Zylinder, AF Aluminium-Folie, $\mathrm{P}$ Präparate, zHP zur Hochvakuumspumpe, F Fenster, L Lumineszenzlicht zum SEV.

der auf die Bestrahlungskammer floß, mit dem Elektronenstrom über einen kleinen Faraday-Käfig, der an Stelle des Präparates eingesetzt werden konnte. Die Fluoreszenzspektren wurden mit einem LeitzMonochromator und dem genannten Sekundärelektronenverstärker gemessen. Die zur UV-Anregung benutzte Lichtquelle war eine Quecksilberhochdrucklampe mit vorgeschaltetem Schott UG 11-Filter.

\section{Herstellung der Präparate}

1. Polystyrolfolien mit Zusatz des Fluoreszenzstoffes nach der Polymerisation (Folie I)

Es wurden mit bekannten Konzentrationen des Fluoreszenzstoffes und von Polystyrol in Xylol zwei Lösungen angesetzt und im gewünschten Verhältnis miteinander gemischt. Die nicht zu dickflüssige Mischung wurde auf Glasplatten ausgegossen und einige Tage an der Luft getrocknet. Durch die Verdampfung des Lösungsmittels entstanden klare, blasenfreie Polystyrolfolien, die sich leicht von der Glasplatte ablösen ließen. Bei der Verwendung von Lösungsmitteln mit höherem oder niedrigerem Dampfdruck (für Xylol 10 Torr bei Zimmertemperatur) wurden die Folien im

${ }^{1}$ J. W ei B, Nature 153, 748 [1944].

2 E. J. Henley u. A. Miller, Nucleonics 9/6, 62 [1951]. ersten Fall wegen der zu schnellen Verdampfung des Lösungsmittels blasig, im zweiten Fall dauerte die Trocknung erheblich länger. Die Folienstärke war kleiner als $0,5 \mathrm{~mm}$.

2. Polystyrolfolien mit Zusatz des Fluoreszenzstoffes vor der Polymerisation (Folie II)

Lösungen des Fluoreszenzstoffes bekannter Konzentration in unstabilisiertem monomeren Styrol wurden in zugeschmolzenen Ampullen mehrere Tage auf $100^{\circ} \mathrm{C}$ erhitzt, wobei die Lösung polymerisierte. Das Polymerisat wurde nach Zerschlagen der Ampullen in Xylol gelöst und wie unter 1. als Folie ausgegossen.

\section{Die Fluoreszenz von Anthracen-Polystyrol-Folien}

Nach Absorptions- und Emissionsmessungen an Polystyrol-Folien mit Anthracen-Einlagerung vor der Polymerisation, die im hiesigen Institut durchgeführt wurden, scheint die Anthracenemission mit wachsender Konzentration noch $\mathrm{zu}$ steigen, wenn die UV-Absorption bereits $100 \%$ erreicht hat. Bei Untersuchungen zur Klärung dieser Ergebnisse zeigte sich, daß das Fluoreszenzspektrum der Anthracen-Einlagerung wesentlich von der Art der Folienherstellung abhängt, je nachdem der Fluoreszenzstoff vor oder nach der Polymerisation eingelagert wurde. Die Untersuchung der Änderung der Fluoreszenzspektren schien auch wichtig, weil bei der Polymerisation durch Aufspaltung der Doppelbindung des Vinylradikals $\left(-\mathrm{CH}=\mathrm{CH}_{2}\right.$ ) Radikale entstehen, die in irgendeiner Weise auf gelöste Substanzen einwirken können. Wie bei der Bestrahlung wäßriger und nicht wäßriger Lösungen ${ }^{1,2}$ ist in Polystyrol andererseits auch die Möglichkeit der Bildung von Radikalen infolge des Elektronenbeschusses gegeben. So wurden an verschiedenen Kunststoffen, unter ihnen Polystyrol, Vernetzungsreaktionen festgestellt ${ }^{3}$. Es ist daher nicht ausgeschlossen, daß bei der Einwirkung von Elektronen auf Polystyrol-Folien ähnliche Vorgänge wie bei der Polymerisation ablaufen, was in der Tat festgestellt werden konnte.

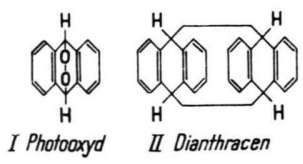

Anthracen wird bereits durch UV-Bestrahlung chemisch verändert. Es kann sich dabei bei Anwesenheit von Sauerstoff das Photooxyd (I) oder in Lösungen Dianthracen (Para-Anthracen) (II) bilden ${ }^{4}$.

${ }^{3}$ A. Charlesby, Nature 171, 167 [1953].

${ }^{4}$ E. Clar, Aromatische Kohlenwasserstoffe, Springer, Berlin 1952, S. $178-179$. 
In Lösungen besitzt Anthracen ein charakteristisches Fluoreszenzspektrum mit zum Teil scharf ausgeprägten Maxima. Die Lage der Maxima ist von der Art des Lösungsmittels abhängig und kann durch folgende Formel $^{5}$ wiedergegeben werden:

$$
\bar{v}=\bar{v}_{0}-1380 \cdot v \text {. }
$$

Darin ist: $\bar{v}$ Wellenzahl des Emissionsmaximums, $\bar{v}_{0}$ eine konstante Wellenzahl, die vom Lösungsmittel abhängig ist; $v=0 ; 1 ; 2 ; 3 \ldots$.

Die in Gl. (1) auftretende Zahl ,1380“ entspricht der Eigenfrequenz der beim Elektronenübergang bevorzugt angeregten Schwingungsform im Grundzustand.

1. Die Fluoreszenzspektren von AnthracenEinlagerung in Polystyrol nach der Polymerisation (Folie I)

Die Fluoreszenzspektren von Anthracen in Polystyrol sind in Abb. 2 wiedergegeben. Sie wurden an Folien aufgenommen, denen Anthracen in Konzentrationen von $0,065-3 \mathrm{~g} / 100 \mathrm{~g}$ nach der Polymeri-

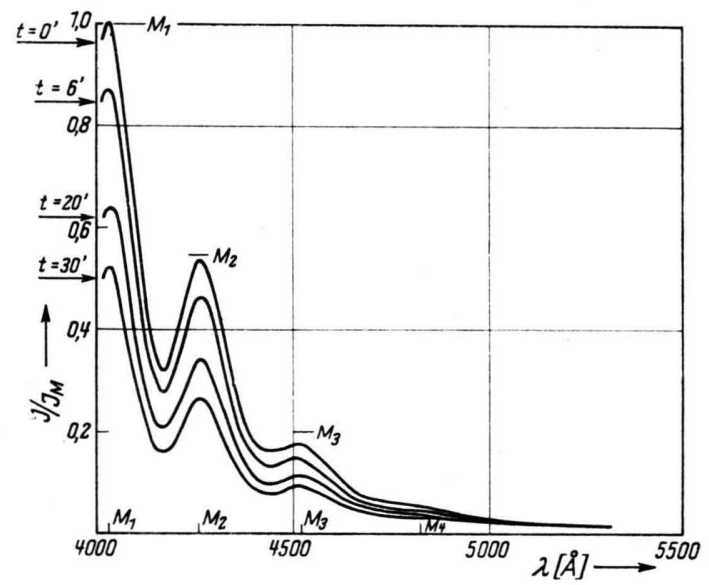

Abb. 2. Fluoreszenzspektrum einer Polystyrolfolie I mit Anthracen bei UV-Anregung (Einlagerung nach der Polymerisation); Konzentration: $2 \mathrm{~g} / 100 \mathrm{~g}$; Bestrahlungszeiten (UV): $t=0,6,20$ und $30 \mathrm{~min}$. Ordinate: $J / J_{\mathbf{M}_{1}}$.

sation (Folie I) zugesetzt wurde. Für die Durchmessung des Fluoreszenzspektrums wurde eine Zeit von 3-4 Minuten benötigt. Da die Fluoreszenzintensität in diesem Zeitraum bereits erheblich absank, wurden zwei Gruppen von Messungen durchgeführt. Zuerst wurde das Fluoreszenzspektrum in Abhängigkeit von der Zeit aufgenommen, wobei das

5 Th. Förster, Fluoreszenz organischer Substanzen, Vandenhoek u. Ruprecht, Göttingen 1951, S. 134.
Spektrum stets von kürzeren nach längeren Welienlängen durchlaufen wurde. In einer zweiten Meßreihe wurde nur die Intensität der Maxima bestimmt, wofür eine Zeit von etwa 10 sec benötigt wurde, eine Zeitspanne, in der die Abnahme der Fluoreszenzintensität vernachlässigbar war. Die Spektrenaufnahmen wurden an Folien durchgeführt, die eine Stärke von $3 \cdots 5 \cdot 10^{-2} \mathrm{~mm}$ besaßen. In Abb. 2 wurde als Ordinatenmaßstab das Verhältnis $J / J_{\mathrm{M}_{1}}$, der jeweiligen Intensität $J$ zur Intensität $J_{\mathbf{M}_{1}}$ des ersten Maximums $\mathbf{M}_{1}$, bei Beginn der Bestrahlung aufgetragen. Die Intensität der Maxima zur Zeit $t=0$ (2. Meßreihe) ist mit der Bezeichnung $\mathbf{M}_{i}(i=$ Nummer des Maximums) in Abb. 2 eingetragen (vgl. auch Abb. 3-5). Es lassen sich vier verschiedene Maxima unterscheiden:

\begin{tabular}{|c|c|c|c|c|}
\hline & $\mathrm{M}_{1}$ & $\mathrm{M}_{2}$ & $\mathrm{M}_{3}$ & $\mathrm{M}_{4}$ \\
\hline$\lambda_{(1-4)}[\AA]$ & $4025-4035$ & 4260 & 4525 & 4800 \\
\hline
\end{tabular}

Ihre Lage ist im Konzentrationsbereich von 0,055 $3 \mathrm{~g} / 100 \mathrm{~g}$ praktisch unabhängig von der Konzentration.

Das Intensitätsverhältnis der Fluoreszenzmaxima ist ebenfalls praktisch unabhängig von der Konzentration

$$
\left(A=J_{\lambda_{1}} / J_{\lambda_{2}}=1,9 ; B=J_{\lambda_{2}} / J_{\lambda_{3}}=2,8\right) .
$$

Der Charakter des Fluoreszenzspektrums bleibt erhalten, wenn mit wachsender UV-Bestrahlungsdauer die Fluoreszenzintensität sinkt.

2. Die Fluoreszenzspektren von AnthracenEinlagerung in Polystyrol vor der Polymerisation (Folie II)

Die Fluoreszenzspektren von Folien, denen Anthracen vor der Polymerisation zugesetzt wurde (Folie II), zeigen ein vom normalen Anthracenlösungsspektrum abweichendes Bild. Es ist schon besonders auffallend, daß selbst bei einer Konzentration von $10 \mathrm{~g} / 100 \mathrm{~g}$ Anthracen in Polystyrol kein Ausfallen von Kristallen beobachtet wurde, während bei der Trocknung der Folien I Anthracen bereits oberhalb einer Konzentration von $3 \mathrm{~g} / 100 \mathrm{~g}$ als Makrokristalle auszufallen beginnt. Die erhöhte Löslichkeit von Anthracen in Folie II könnte ihre Ursache in der hohen Polymerisationstemperatur von $100^{\circ} \mathrm{C}$ haben. Bei der Abkühlung des Polymerisats auf Zimmertemperatur würde die Auskristallisation von Makrokristallen infolge der Zähigkeit der Substanz vielleicht verhindert sein. Wäre diese Annahme richtig, müßten bei der Lösung dieses Poly- 
merisats in Xylol und anschließender Herstellung der Polystyrolfolie Makrokristalle ausfallen. Ihre Bildung wurde jedoch nicht beobachtet.

Diese Erscheinung legt schon die Vermutung nahe, daß bei der Polymerisation Veränderungen am eingelagerten Anthracen vor sich gegangen sein müssen. Diese Vermutung wird durch die Veränderungen im Anthracen-Spektrum bestätigt.

Das Fluoreszenzspektrum einer Polystyrolfolie mit 10/100g Anthracen (Abb. 3) zeichnet sich durch drei gut ausgeprägte Maxima $M_{5}-M_{7}$ aus. Daneben ist noch ein schwaches Maxima $M_{8}$ bei etwa $4690 \AA$ angedeutet:

\begin{tabular}{|c|c|c|c|c|}
\hline & $\mathrm{M}_{5}$ & $\mathrm{M}_{6}$ & $\mathrm{M}_{7}$ & $\mathrm{M}_{8}$ \\
\hline$\lambda_{(5-8)}[\AA]$ & 4025 & 4160 & 4405 & 4690 \\
\hline
\end{tabular}

Mit wachsender UV-Bestrahlung nimmt die Intensität des Spektrums ab. Besonders auffallend ist die starke Schwächung des Maximums $\mathrm{M}_{6}$ bei $4160 \AA$. Die Maxima $\mathrm{M}_{6}-\mathrm{M}_{8}$ sind nach einer Bestrahlungszeit von $60 \mathrm{~min}$ verschwunden, dafür haben sich aber neue Maxima $\mathrm{M}_{9}$ und $\mathrm{M}_{10}$ ausgebildet:

\begin{tabular}{|c|c|c|}
\hline & $\mathrm{M}_{9}$ & $\mathrm{M}_{20}$ \\
\hline$\lambda_{(9-10)[\AA]}$ & 4260 & 4525 \\
\hline
\end{tabular}

Mit sinkender Anthracenkonzentration nimmt die Intensität des Maximums $\mathrm{M}_{6}$ gegenüber dem Maximum $\mathrm{M}_{5}$ stark ab (Abb. 3-5). Je niedriger die Anthracenkonzentration wird, um so mehr ist das Maximum $\mathrm{M}_{9}$ schon zu Beginn der Bestrahlung angedeutet. Bei der Folie mit 0,1 g/100g Anthracen in Polystyrol sind die Fluoreszenzmaxima $\mathrm{M}_{6}$ und

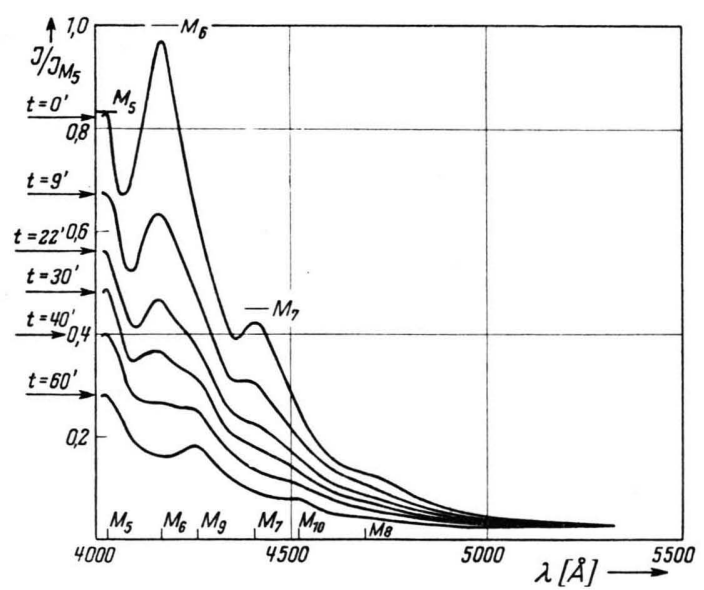

Abb. 3. Fluoreszenzspektrum einer Polystyrolfolie II mit Anthracen bei UV-Anregung (Einlagerung vor der Polymerisation); Konzentration $10 \mathrm{~g} / 100 \mathrm{~g}$; Bestrahlungszeiten (UV): $t=0,9,22,30,40$ und $60 \mathrm{~min}$.
$M_{7}$ vollständig verschwunden, es treten nur die Maxima $M_{5}, M_{9}$ und $M_{10}$ auf.

Die Lage der Fluoreszenzmaxima ist unabhängig von der Konzentration.

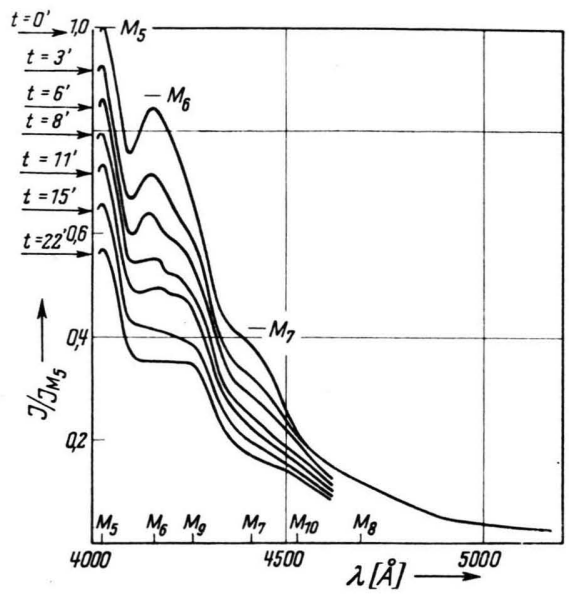

Abb. 4. Fluoreszenzspektrum einer Polystyrolfolie II mit Anthracen bei UV-Anregung (Einlagerung vor der Polymerisation); Konzentration: $2 \mathrm{~g} / 100 \mathrm{~g}$; Bestrahlungszeiten (UV): $t=0,3,6,8,11,15$ und $22 \mathrm{~min}$.

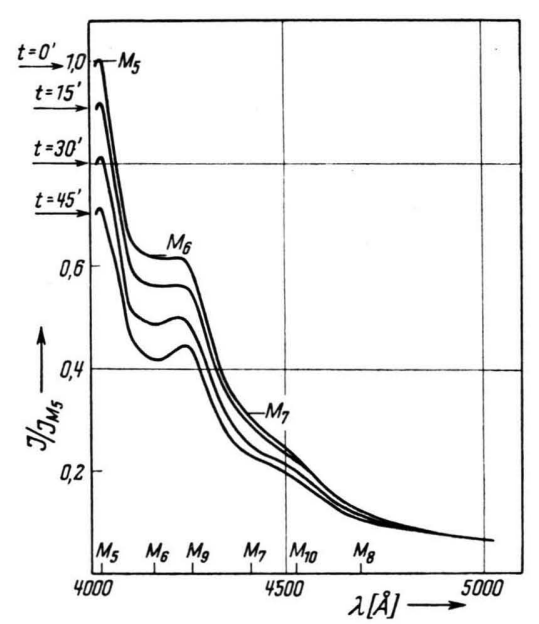

Abb. 5. Fluoreszenzspektrum einer Polystyrolfolie II mit Anthracen bei UV-Anregung (Einlagerung vor der Polymerisation); Konzentration: $0,1 \mathrm{~g} / 100 \mathrm{~g}$; Bestrahlungszeiten: (UV): $t=0,15,30$ und $45 \mathrm{~min}$.

Das Verhältnis der Intensitäten der Maxima $\mathbf{M}_{5}$ $\mathrm{zu} \quad \mathrm{M}_{6}$ und $\mathrm{M}_{6} \mathrm{zu} \mathrm{M}_{7}\left(C=J_{\mathrm{M}_{5}} / J_{\mathrm{M}_{6}} ; D=J_{\mathrm{M}_{6}} / J_{\mathrm{M}_{7}}\right)$ in Abhängigkeit von der Konzentration ist in Tab. 1 eingetragen.

Die Werte wurden in der gleichen Weise wie bei den Folien I gemessen. Während der Wert des Verhältnisses $C$ von 0,83 bei einer Konzentration von $10 \mathrm{~g} / 100 \mathrm{~g}$ bis 1,61 bei $0,1 \mathrm{~g} / 100 \mathrm{~g}$ mit sinkender Kon- 
zentration beträchtlich zunimmt, scheint der Wert des Verhältnisses $D$ nur ein wenig innerhalb des genannten Konzentrationsbereiches zu fallen. Bei der Folie mit 0,1 g/l00g Anthracen in Polystyrol wurde dabei die Intensität der Wellenlänge zur Bildung der Maximaverhältnisse $C$ und $D$ benutzt, die zu diesen Maxima gehört. Die starke Zunahme des Verhältnisses $C$ mit sinkender Konzentration einerseits und die praktische Konstanz des Verhältnisses $D$ weisen darauf hin, daß die Maxima $\mathbf{M}_{6}$ und $\mathbf{M}_{\mathbf{7}}$ einander zugeordnet sind.

\begin{tabular}{|c|rrr|rr|}
\hline \multirow{2}{*}{$\begin{array}{c}\text { Anthracen- } \\
\text { konzentration } \\
\text { in [g } / 100 \mathrm{~g}]\end{array}$} & \multicolumn{4}{|c|}{$\begin{array}{c}\text { Relative Intensität } \\
\text { der Wellenlängen } \\
\lambda_{5}\end{array} \lambda_{\lambda_{6}}$} & \multicolumn{2}{|c|}{$\begin{array}{c}\text { Intensitätsverhältnis } \\
\lambda_{7}\end{array}$} & $J_{\lambda_{5}} / J_{\lambda_{6}} B=J_{\lambda_{6}} J_{\lambda_{7}}$ \\
\hline 10 & 100 & 120 & 55,5 & 0,83 & 2,16 \\
8 & 100 & 119 & 56,5 & 0,84 & 2,10 \\
7 & 100 & 119 & 56 & 0,84 & 2,12 \\
6 & 100 & 114 & 53,5 & 0,88 & 2,13 \\
3 & 100 & 92 & 44 & 1,09 & 2,09 \\
2 & 100 & 87 & 41,5 & 1,15 & 2,10 \\
1 & 100 & 76 & 35 & 1,32 & 2,16 \\
0,1 & 100 & 63 & 31,2 & 1,61 & 2,02 \\
\hline
\end{tabular}

Tab. 1. Relative Intensität der Fluoreszenzmaxima in AnthracenPolystyrolfolien II.

3. Der Vergleich der Fluoreszenzspektren der Folien I und II

Wenn man die Fluoreszenzspektren der Folien I (Zusatz nach der Polymerisation) und der Folien II (Zusatz vor der Polymerisation) bei gleicher Konzentration miteinander vergleicht, erhält man interessante Ergebnisse.

In den Abb. 6-8 wurden die Fluoreszenzspektren der Folien I und II mit Anthracenkonzentrationen von $2 ; 1 ; 0,1 / 100 \mathrm{~g}$ mit den bereits früher benutzten Ordinaten-Maßstäben $J / J_{\mathbf{M}_{1}}$ bzw. $J / J_{\mathbf{M}_{5}}$

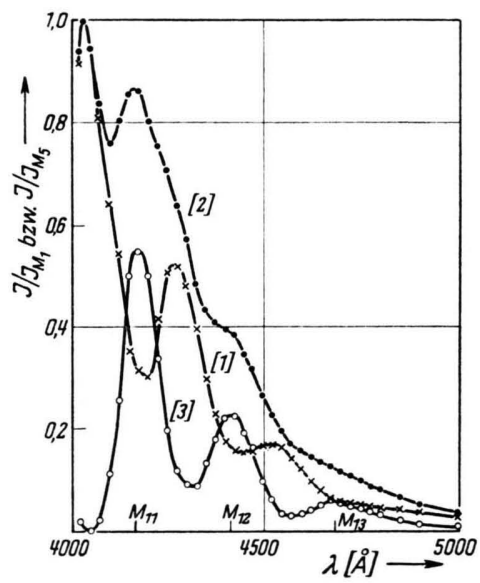

Abb. 6. Fluoreszenzspektren von Anthracen-Polystyrolfolien. Konzentration: $2 \mathrm{~g} / 100 \mathrm{~g}$.

[1] Folie I (Einlagerung nach der Polymerisation),

[2] Folie II (Einlagerung vor der Polymerisation), [3] Differenzspektrum von [1] und [2]. übereinander gezeichnet. Die Fluoreszenzmaxima $\mathrm{M}_{1}$ und $\mathrm{M}_{5}$ fallen daher zusammen (Spektrum [1] entspricht Folie I, Spektrum [2] entspricht Folie II). Bildet man nun die Differenz zwischen beiden

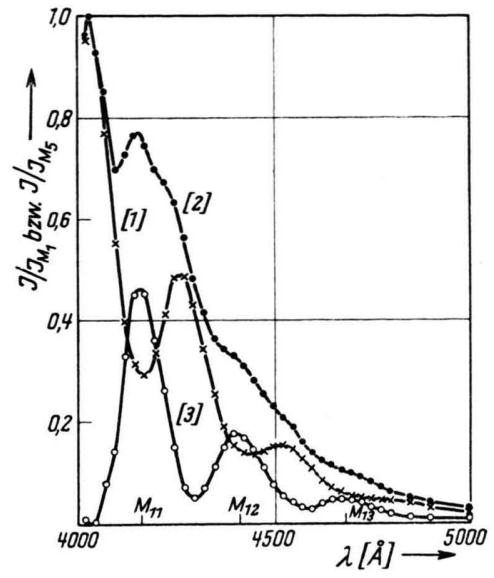

Abb. 7. Fluoreszenzspektren von Anthracen-Polystyrolfolien. Konzentration: $1 \mathrm{~g} / 100 \mathrm{~g}$.

[1] Folie I (Einlagerung nach der Polymerisation), [2] Folie II (Einlagerung vor der Polymerisation), [3] Differenzspektrum von [1] und [2].

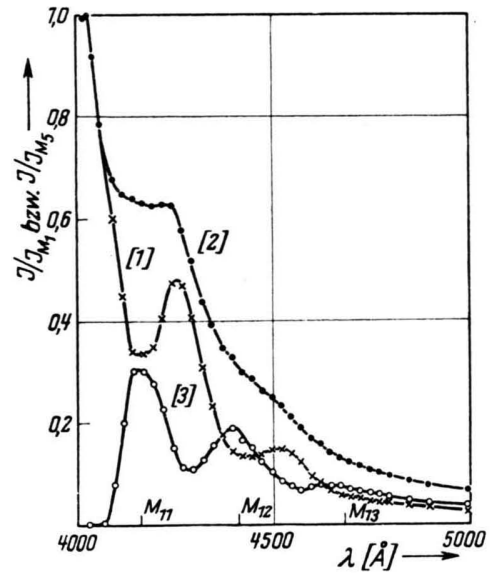

Abb. 8. Fluoreszenzspektren von Anthracen-Polystyrolfolien. Konzentration: $0,1 \mathrm{~g} / 100 / \mathrm{g}$.

[1] Folie I (Einlagerung nach der Polymerisation), [2] Folie II (Einlagerung vor der Polymerisation), [3] Differenzspektrum von [1] und [2].

Fluoreszenzspektren, so erhält man als Resultat das Spektrum [3]. Dieses Differenzspektrum besitzt bei allen Konzentrationen drei Maxima $M_{11}-M_{13}$.

\begin{tabular}{|c|c|c|c|}
\hline & $\mathrm{M}_{11}$ & $\mathrm{M}_{12}$ & $\mathrm{M}_{13}$ \\
\hline$\lambda_{(11-13)[\AA]}$ & 4160 & 4405 & 4680 \\
\hline
\end{tabular}

In Tab. 2 sind diese Maxima mit den bereits gefundenen Maxima $\mathrm{M}_{1}-\mathrm{M}_{10}$ zusammengestellt. 
Der Vergleich der einzelnen Spektren miteinander legt die Vermutung nahe, daß sich in der Folie II zwei Spektren $S_{1}$ und $S_{2}$ überlagern:

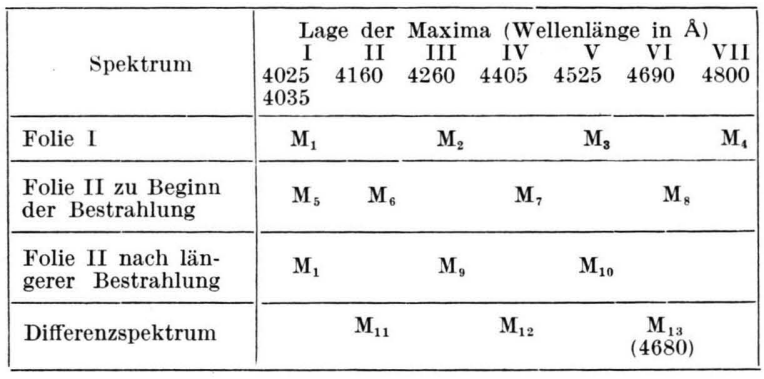

Tab. 2.

a) das Spektrum $S_{1}$, das dem Spektrum der Folie I entspricht, mit den Maxima $\mathrm{M}_{\mathrm{I}} ; \mathrm{M}_{\mathrm{III}} ; \mathrm{M}_{\mathrm{V}}$ und $\mathrm{M}_{\mathrm{VII}}$. (Die Bezeichnung lehnt sich an Tab. 2 an.)

b) das Spektrum $\mathrm{S}_{2}$ mit den Maxima $\mathrm{M}_{\mathrm{II}} ; \mathrm{M}_{\mathrm{IV}}$; $\mathrm{M}_{\mathrm{VI}}$, das dem Spektrum $\mathrm{S}_{1}$ qualitativ gleich ist, aber nach längeren Wellenlängen verschoben erscheint.

Damit stimmt die Feststellung überein, daß das Verhältnis $D$ der Maxima $\mathrm{M}_{6}$ und $\mathrm{M}_{7}\left(\mathrm{M}_{\mathrm{II}} ; \mathrm{M}_{\mathrm{IV}}\right)$ praktisch unabhängig von der Anthracenkonzentration ist, woraus bereits früher auf eine Zuordnung der Maxima zueinander geschlossen wurde. Setzt man die Wellenzahl der Maxima der Spektren $\mathrm{S}_{1}$ und $\mathrm{S}_{2}\left(\mathrm{M}_{\mathrm{I}}: \lambda_{1}=4025 \AA\right)$ in Gl. (1) ein, so erhält man mit $v=1 ; 2 ; 3$ (das Maximum mit $v=0$ liegt im UV-Bereich) für $\bar{v}_{0}$ folgende ungefähren Werte:

$$
\begin{aligned}
& \text { Spektrum } \mathrm{S}_{1}: \overline{v_{0}}=26230 \mathrm{~cm}^{-1}, \\
& \text { Spektrum } \mathrm{S}_{2}: \overline{v_{0}}=25450 \mathrm{~cm}^{-1} .
\end{aligned}
$$

Da die Lage der Maxima nur mit einem Fehler von etwa $10 \AA$ geschätzt werden konnte, stellen die Werte für die Wellenzahlen nur Mittelwerte dar.

Unter Berücksichtigung der relativ geringen Meßgenauigkeit der Wellenlänge der Spektrenmaxima erfüllen ihre Wellenzahlen mit den genannten Werten für $\overline{v_{0}}$ Gl. (1) recht gut. Die Existenz der Anthracenspektren $S_{1}$ und $S_{2}$ dürfte daher hinreichend sicher sein.

Bei der UV-Anregung sinkt die Intensität des verlagerten Anthracenspektrums $\mathrm{S}_{2}$ rascher als bei dem nicht verlagerten $S_{1}$, so daß mit wachsender Bestrahlungszeit die Maxima des letzteren $\left(\mathrm{S}_{1}\right)$ auch bei der Folie II erkennbar werden, während sie vorher in der großen Intensität des $\mathrm{S}_{2}$-Spektrums verschwanden.
Diesen Anthracenspektren überlagert sich noch das ,Eigenspektrum“ von Polystyrol. Nach Koski ${ }^{6}$ besitzt reines Polystyrol ein Fluoreszenzspektrum mit einer langwelligen Grenze bei etwa $3800 \AA$. Das bei den Untersuchungen dieser Arbeit benutzte Polystyrol emittiert jedoch eine schwache, violette Fluoreszenz bei UV-Anregung, die vermutlich durch Verunreinigungen hervorgerufen wird. (Maximum im sichtbaren Bereich bei 4050 A.) Die Intensität der Polystyrol-Fluoreszenz dürfte auf den Charakter der Spektren der Folien I und II oberhalb $4000 \AA$ bei genügend hoher Anthracenkonzentration nur einen geringen Einfluß haben. Sie beträgt nämlich bei $4025 \AA$, dem Maximum $\mathrm{M}_{1}$, im Vergleich zur Intensität der Folie I mit einer Anthracenkonzentration von $2 \mathrm{~g} / 100 \mathrm{~g}$ nur $4,2 \%$.

Aus den vorliegenden Ergebnissen wird deutlich, daß bei der Polymerisation von monomerem Styrol mit Anthracenbeimengung ein Teil der Anthracenmoleküle, wegen der besonders hohen Leuchtintensität des Spektrums $S_{2}$ vielleicht der größte Teil, in einen veränderten Zustand überführt wird.

\section{Die Deutung der Unterschiede der} Fluoreszenzspektren der Folien I und II

Der Kettenabbruch bei der Polymerisation erfolgt offenbar bei der Lösung von Anthracen in Styrol und anschließender Polymerisation zum Teil durch Anthracen-Moleküle. Dabei dürfte die Wahrscheinlichkeit des Kettenabbruchs durch Anthracen-Moleküle eine Funktion ihrer Konzentration sein. Welche Reaktionen dabei ablaufen, läßt sich nicht ohne weiteres sagen. Da das Anthracenspektrum zwar nach längeren Wellenlängen verschoben wird, sein Charakter aber im wesentlichen erhalten bleibt, liegt die Vermutung nahe, daß der Anthracen-Kern nicht verändert wird. Vielleicht wird ein Wasserstoffatom abgespalten und durch ein Polystyrolradikal ersetzt.

In diesem Zusammenhang wird auch verständlich, daß sich mindestens $10 \mathrm{~g} / 100 \mathrm{~g}$ Anthracen in Polystyrolfolien einbetten lassen, während das Anthracen in der Folie I oberhalb einer Konzentration von $3 \mathrm{~g} / 100 \mathrm{~g}$ bereits auszufallen beginnt. Der größte Teil, mindestens aber $7 \mathrm{~g} / 100 \mathrm{~g}$, muß daher chemisch verändert worden sein. Die Abnahme des Verhältnisses $C$ der Intensitäten der Maxima $\mathrm{M}_{5}$ und $\mathrm{M}_{6}$ in Folie II mit wachsender Konzentration scheint auch dafür zu sprechen, daß der größte Teil des zu-

$$
{ }^{6} \text { W. S. Koski, Phys. Rev. 82, } 230 \text { [1951]. }
$$


gegebenen Anthracens in eine andere Verbindung überführt sein dürfte, welche das noch verbliebene Anthracen an Fluoreszenzintensität stark überwiegt. Daß diese Verbindung nicht sehr stabil ist, und daher bei der UV-Anregung leicht zerfällt, ist durchaus denkbar.

Die Veränderung des Anthracens kann allerdings nicht allein beim Kettenabbruch erfolgt sein. Wäre nämlich der größte Teil der Anthracen-Moleküle an den Kettenenden gebunden, so müßte z. B. bei einer Konzentration von $10 \mathrm{~g} / 100 \mathrm{~g}$ die Zahl der Kettenmoleküle so groß sein, daß sie nur aus weniger als 100 Grundbausteinen bestehen könnten. Dies ist aber offenbar nicht der Fall, da die Härte des Polymerisats auf eine wesentlich größere Kettenlänge schließen läßt.

Die Deutung der Ergebnisse bei der UV-Anregung von Anthracen-Polystyrolfolien wird durch Beobachtungen bei der Kathodenstrahlanregung erhärtet. Es entsteht dabei nämlich in Folie I unter der Einwirkung der Elektronen das verlagerte Spektrum $\mathrm{S}_{2}$. Anthracen wird durch den Elektronenbeschuß in einen Zustand überführt, der auch durch die Polymerisation erzeugt wird. Die Versuchsergebnisse werden im nächsten Abschnitt mitgeteilt.

5. Die Fluoreszenz von Anthracen-Polystyrolfolien unter der Einwirkung von Elektronen

In Vorversuchen wurde festgestellt, daß Polystyrol-Präparate sich unter dem Elektronenbeschuß stark aufluden. Um diese Aufladungseffekte zu verhindern, wurden die Folien auf der Vorderseite mit einer Silberschicht von durchschnittlich $0,7 \cdot 10^{-3} \mathrm{~mm}$ im Vakuum bedampft. Diese Silberschicht war dünn genug, um noch genügend Fluoreszenzintensität zur Messung hindurchzulassen.

Die bei der Kathodenstrahlanregung benutzten Folien hatten in der Regel eine Stärke von wenigen $10^{-1} \mathrm{~mm}$. Unter Verwendung der praktischen Reichweite von Elektronen in Aluminium ${ }^{7}$, die bei $40 \mathrm{keV}$ etwa $3 \mathrm{mg} / \mathrm{cm}^{2}$ beträgt, errechnet sich die Reichweite der Elektronen in Polystyrol bei einer Dichte von $1,05 \mathrm{~g} / \mathrm{cm}^{3} \mathrm{zu}$ etwa $3 \cdot 10^{-3} \mathrm{~mm}$. Wegen der geringen Eindringtiefe der Elektronen war die genaue Bestimmung der Folienstärke nicht erforderlich. Selbst wenn man berücksichtigt, daß Reabsorption der Pri1933. märemission und anschließende Emission in der Schicht jenseits der Reichweite der Elektronen auftreten kann, dürften Dickeschwankungen nur einen geringen Einfluß auf das Meßergebnis haben.

Im Gegensatz zu der von Hengst gemessenen Intensitätsabnahme der Fluoreszenz von kristallinem Anthracen bei der Kathodenstrahlanregung, 8 , wurde in Anthracen-Polystyrolfolien Intensitätszunahme beobachtet. Abb. 9 zeigt die Ab-

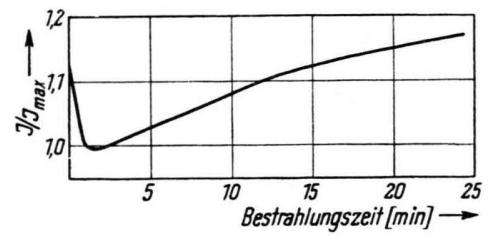

Abb. 9. Fluoreszenzintensität einer Anthracen-Polystyrolfolie I in Abhängigkeit von der Bestrahlungszeit bei Kathodenstrahlanregung.

hängigkeit der Fluoreszenzintensität einer Folie mit einer Anthracen-Konzentration von $2 \mathrm{~g} / 100 \mathrm{~g}$ von der Bestrahlungszeit bei Kathodenstrahlanregung. Die Beschleunigungsspannung der Elektronen betrug $50 \mathrm{kV}$. Der Elektronenstrom am Präparat hatte eine Dichte von $9,4 \cdot 10^{-8} \mathrm{Amp} / \mathrm{cm}^{2}$. Zur Messung der Fluoreszenzintensität wurde das Fluoreszenzlicht nicht gefiltert, um genügend Lichtintensität zur Messung zu erhalten.

Die anfängliche Intensitätsabnahme in Abb. 9 ist vermutlich durch Ausbildung einer Raum-

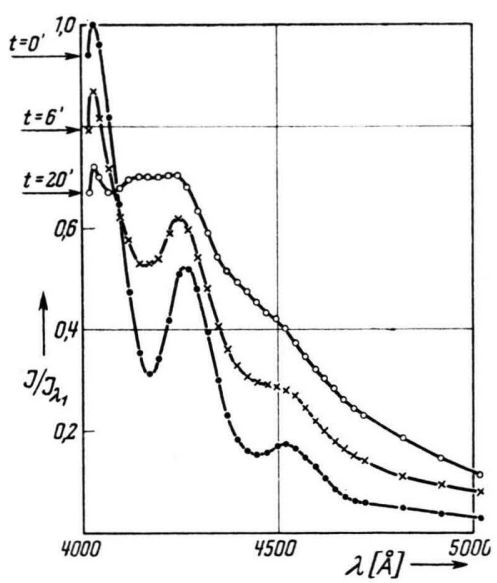

Abb. 10. Fluoreszenzspektrum einer Anthracen-Polystyrolfolie I bei UV-Anregung. Konzentration: $2 \mathrm{~g} / 100 \mathrm{~g}$. Vorhergehende Bestrahlung mit Kathodenstrahlen, Dauer: $t=0,6$ und $20 \mathrm{~min}$.

${ }^{8}$ K. Hengst, Z. Naturforschg. 6a, 540 [1951].

${ }^{9}$ W. Hanle u. K. H. Rau, Z. Phys. 133, 297 [1952]. 
ladung im Präparat infolge der in die Folie eingedrungenen Elektronen bedingt. Weitere Ausführungen darüber werden im II. Teil gegeben.

Zur Klärung der nicht erwarteten Zunahme der Gesamtfluoreszenzintensität wurde das Spektrum bei UV-Anregung von bestrahlten Folien mit dem der unbestrahlten Folien verglichen. Abb. 10 gibt die Spektren wieder. Für diese Messung wurden Folien mit einer Stärke von $4,5 \cdot 10^{-3} \mathrm{~cm}$ hergestellt. Damit die eingeschossenen Elektronen die Folie in ihrer ganzen Tiefe durchsetzten, wurden diese von beiden Seiten bestrahlt. Die Spektren sind mit dem Ordinaten-Maßstab $J / J_{\lambda_{1}}$ wiedergegeben, dabei ist $J_{\lambda_{1}}$ die Intensität des ersten Maximums der Folie vor der Kathodenstrahlanregung. Nimmt man als Ordinaten-Maßstab $J / J_{0}$, worin $J_{0}$ die Intensität des jeweiligen Spektrums bei der Wellenlänge $\lambda_{1}=$ $4025 \AA$ ist, und bildet die Differenz der Spektren, so erhält man das Spektrum [3] in Abb. 11.

Dieses Differenzspektrum besitzt drei Maxima:

\begin{tabular}{|c|c|c|c|}
\hline & $\mathrm{M}_{14}$ & $\mathrm{M}_{15}$ & $\mathrm{M}_{11}$ \\
\hline$\lambda_{(14-16)}[\AA]$ & 4170 & 4400 & 4660 \\
\hline
\end{tabular}

Sie liegen bis auf geringe Abweichungen an den Stellen der Maxima $\mathrm{M}_{\mathrm{II}}, \mathrm{M}_{\mathrm{IV}}$ und $\mathrm{M}_{\mathrm{VI}}$ der Tabelle 2 und entsprechen also den Maxima des nach längeren Wellenlängen verschobenen Anthracenspektrums $\mathrm{S}_{2}$.

Das Auftreten dieses Spektrums bei der Kathodenstrahl-Anregung von Polystyrolfolien I führt zu der Schlußfolgerung, daß durch den Elektronenbeschuß wenigstens zum Teil dieselben chemischen Veränderungen der Anthracen-Moleküle auftreten, wie sie als Folge der Polymerisation beobachtet wurden. In Abb. 10 erkennt man darüber hinaus das Anwachsen eines kontinuierlichen Untergrundspektrums mit wachsender Kathodenstrahlanregung. Vermutlich sind dafür ebenfalls chemische Umsetzungen verantwortlich, über deren Natur jedoch nichts ausgesagt werden kann.

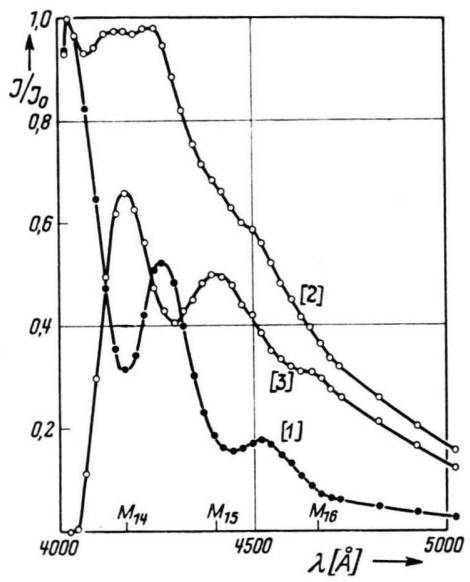

Abb. 11. Fluoreszenzspektren einer Anthracen-Polystyrolfolie I bei UV-Anregung. Konzentration : $2 \mathrm{~g} / 100 \mathrm{~g}$. [1] vor der Kathodenstrahlanregung, [2] nach der Kathodenstrahlanregung, Dauer: $20 \mathrm{~min}$, [3] Differenzspektrum von [1] und [2].

In diesem Zusammenhang seien die bei der Einwirkung energiereicher Strahlung auf Kunststoffe beobachteten Vernetzungsreaktionen erwähnt ${ }^{3}$.

Bei der Bestrahlung der Folien II verändert sich das Fluoreszenzspektrum ebenfalls in starkem Maße. Bei dem Differenzspektrum zeigt sich ein Maximum bei etwa $4300 \AA$, das sich näher zuordnen läßt. 